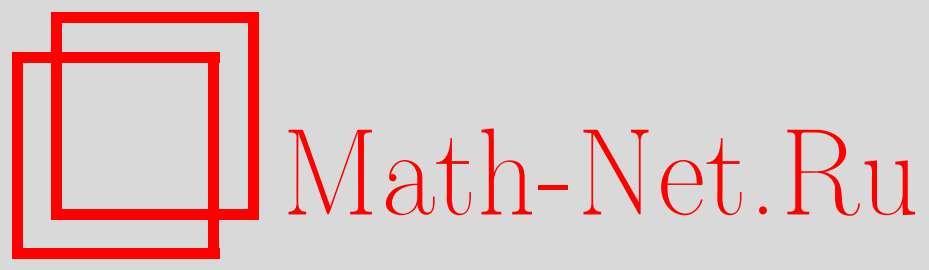

В. В. Жиков, Диффузия в несжимаемом случайном потоке, Функи. анализ и его прил., 1997, том 31, выпуск 3, 10-22

DOI: https://doi.org/10.4213/faa471

Использование Общероссийского математического портала MathNet.Ru подразумевает, что вы прочитали и согласны с пользовательским соглашением

http://www . mathnet.ru/rus/agreement

Параметры загрузки:

IP : 3.82 .47 .9

26 апреля 2023 г., 16:18:02

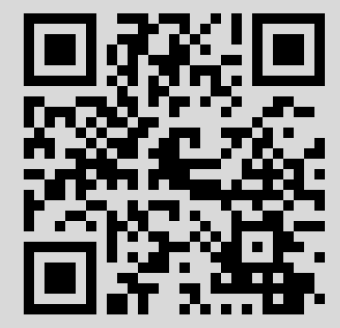


Функииональный анализ и его приложения

1997 , т. 31, вып. 3, с. 10-22

УДК 517.97

\title{
Диффузия в несжимаемом случайном потоке
}

\author{
(c) 1997. В. В. Жииков
}

Памяти Сергея Михайловича Козлова

\section{§1. Введение}

1. Рассмотрим уравнение

$$
\frac{\partial \rho}{\partial t}=\Delta \rho-b(x) \cdot \nabla \rho, \quad x \in \mathbb{R}^{d}, t>0,
$$

в котором $b(x)$ - случайное однородное соленоидальное векторное поле в $\mathbb{R}^{d} \mathrm{c}$ нулевым средним: $\operatorname{div} b(x)=0,\langle b\rangle=0$.

Примером служит периодическое или почти периодическое соленоидальное поле. В этом случае $\langle b\rangle$ есть обычное среднее периодической или почти периодической функции. Уравнению (1.1) подчиняется плотность распределения броуновской частицы в стационарном потоке жидкости. Основной вопрос связан с асимптотическим поведением плотности $\rho(x, t)$ при большом $t$. Если роль конвективного члена $b \cdot \nabla \rho$ сводится к замене исходного уравнения «усредненным» уравнением $\partial \rho / \partial t=\operatorname{div}\left(A^{0} \nabla \rho\right)$, в котором $A^{0}>0-$ постоянная матрица, то говорят о диффузионном поведении частицы.

Удобно переписать уравнение (1.1) в терминах матрицы тока. Для этого представим соленоидальный вектор $b(x)$ в виде дивергенции от кососимметрической матрицы:

$$
b_{i}(x)=\frac{\partial}{\partial x_{j}} B_{i j}(x), \quad B_{i j}=-B_{j i},
$$

что возможно, если, например, $b$ периодичен, $\left\langle|b|^{2}\right\rangle<\infty$. Тогда $b \cdot \nabla \rho=$ $\operatorname{div}(B \nabla \rho)$, и мы получаем задачу Коши для дивергентного уравнения:

$$
\begin{gathered}
\frac{\partial \rho}{\partial t}=\Delta \rho-\operatorname{div}(B \nabla \rho)=\operatorname{div}(\nabla \rho(x) A(x)), \\
\left.\rho\right|_{t=0}=\chi, \quad \chi \geqslant 0, \chi \in L^{2}, \quad \int_{\mathbb{R}^{d}} \chi d x=1,
\end{gathered}
$$

где $A(x)=E+B(x), E$ - единичная матрица. Например, в случае $d=2$

$$
b_{1}=\frac{\partial \alpha}{\partial x_{2}}, \quad b_{2}=-\frac{\partial \alpha}{\partial x_{1}}, \quad B=\left(\begin{array}{cc}
0 & \alpha \\
-\alpha & 0
\end{array}\right) .
$$

\footnotetext{
^ Работа выполнена при финансовой поддержке РФФИ (проект №96-01-00503).
} 
Мы покажем, что если $b(x)$ и $B(x)$ - реализации квадратично интегрируемых случайных однородных полей, то имеет место диффузионное поведение. Соответствующая чентральная предельная теорема (ц.п.т.) будет получена как следствие подходящих свойств усреднения. Примеры показывают, что условие квадратичной интегрируемости, $\left\langle|B|^{2}\right\rangle<\infty$, необходимо для конечности матрицы эффективной диффузии и тем более для других свойств усреднения. Здесь нужно принять во внимание следующие факты.

(i) Несжимаемость потока всегда ведет к увеличению диффузии: $A^{0} \geqslant E$, см. [1, с. $30 ; 2$, с. 23], а также $\S 2$ настоящей работы.

(ii) Если $\left\langle|B|^{2}\right\rangle=\infty$, то матрича $A^{0}$ может оказаться бесконечной.

Действительно, рассмотрим двумерный случай, $x=\left(x_{1}, x_{2}\right)$, и пусть $\alpha=$ $\alpha\left(x_{1}\right)$ зависит только от $x_{1}$. Тогда по формуле Зельдовича $[1$, c. $23 ; 2$, с. 16$]$

$$
A^{0}=\left(\begin{array}{cc}
1 & 0 \\
0 & \left\langle\alpha^{2}\right\rangle+1
\end{array}\right) \text {. }
$$

Мы получим бесконечную эффективную диффузию, если $\langle|\alpha|\rangle<\infty,\left\langle\alpha^{2}\right\rangle=\infty$. Конечно, для периодической функции $\alpha\left(x_{1}\right)$ векторное поле $b(x)=\left\{0, \partial \alpha / \partial x_{1}\right\}$ надо понимать в смысле распределений. Но для почти периодических или случайных $\alpha\left(x_{1}\right)$ построены примеры, когда поле $b(x)$ является гладким, см. $[3,4]$.

Часто об исходном векторном поле $b(x)$ «забывают» и имеют дело с уравнением (1.3), в котором кососимметрическая матрица $B(x)$ лишь измерима, тогда говорят о диффузии в турбулентном потоке. Если $B(x)$ ограничена на $\mathbb{R}^{d}$ (будучи периодической, почти периодической или случайной однородной), то все вопросы усреднения и асимптотического поведения хорошо изучены, см. $[1,2$, гл. VII]. В приложениях матрица $B$ обычно не является даже локально ограниченной, а лишь квадратично суммируема. В связи с этим в последние годы обсуждается интересная гипотеза о справедливости теории усреднения при одном лишь условии $\left\langle|B|^{2}\right\rangle<\infty$, см. $[5,6]$. «Технические» трудности, возникающие здесь, можно обозначить термином «проблема единственности». Например, не доказана единственность решения задачи Коши (1.3). Далее, эффективная диффузия $A^{0}$ определяется с помощью эллиптических уравнений на вероятностном пространстве («задачи на ячейке»). Для них также имеются проблемы с единственностью, так что само понятие эффективной диффузии не определено однозначно.

2. Пусть $(\Omega, \mu)$ - вероятностное пространство с $d$-мерной динамической системой $T(x)\left(x \in \mathbb{R}^{d}\right)$, обладающей обычными свойствами:

(i) преобразования $T(x): \Omega \rightarrow \Omega$ сохраняют меру $\mu$,

(ii) для любой $\mu$-измеримой функции $g(\omega)$ на $\Omega$ функция $g(T(x) \omega)$ измерима на $\Omega \times \mathbb{R}^{d}$ (при этом на $\mathbb{R}^{d}$ задается мера Лебега).

Мы предполагаем, что динамическая система $T(x)$ эргодична и используем эргодическую теорему в следующей форме.

ЭРГОДИЧЕСКАЯ ТЕОРЕМА. Пусть $g \in L^{p}(\Omega)=L^{p}(\Omega, d \mu), p \geqslant 1$. Тогдa для $\mu$-почти всех $\omega \in \Omega$ реализачия $g(x)=g(T(x) \omega)$ принадлежит $L_{\mathrm{loc}}^{p}\left(\mathbb{R}^{d}\right)$ $u$ ирu $\varepsilon \rightarrow 0$

$$
g\left(\varepsilon^{-1} x\right) \rightarrow\langle g\rangle=\int_{\Omega} g d \mu \quad \text { слабо в } L_{\mathrm{loc}}^{p}\left(\mathbb{R}^{d}\right)
$$


Допустим, что векторное поле $b(x)$ и кососимметрическая матрица $B(x)$, связанные соотношением (1.2), имеют структуру

$$
b(x)=b(T(x) \omega), \quad B(x)=B(T(x) \omega), \quad b, B \in L^{2}(\Omega) .
$$

Само уравнение диффузии (1.3) имеет смысл для почти каждого $\omega \in \Omega$, а начальное значение $\chi$ считается не зависящим от $\omega$.

Поясним смысл центральной предельной теоремы. По крайней мере в случае $B \in L^{\infty}(\Omega)$ очевидно, что для решения $\rho(x, t, \omega)$ уравнения $(1.3)$

$$
\rho \geqslant 0, \quad \int_{\mathbb{R}^{d}} \rho(x, t, \omega) d x=1 \quad \forall t>0 .
$$

Поэтому функцию $\rho(x, t, \omega)$ можно рассматривать как плотность распределения некоторой случайной величины $\eta(t)=\eta(t, \omega)$. Центральная предельная теорема - это утверждение о том, что случайная величина

$$
\varepsilon \eta\left(\varepsilon^{-2} t\right) \quad(t>0)
$$

при $\varepsilon \rightarrow 0$ сходится к гауссовой случайной величине. Очевидно, что плотностью распределения величины (1.5) будет $\varepsilon^{-d} \rho\left(\varepsilon^{-1} x, \varepsilon^{-2} t, \omega\right)$.

Теорема 1.1 (центральная предельная теорема в среднем). Cуществует постоянная матрича $A^{0}>0$, такая, ито для любых $t \geqslant 0$ u $\varphi \in C_{0}^{\infty}\left(\mathbb{R}^{d}\right)$

$$
\lim _{\varepsilon \rightarrow 0} \int_{\Omega}\left|\int_{\mathbb{R}^{g}}\left[\varepsilon^{-d} \rho\left(\varepsilon^{-1} x, \varepsilon^{-2} t, \omega\right)-K_{0}(x, t)\right] \varphi(x) d x\right| d \mu=0,
$$

где

$$
K_{0}(x, t)=(\pi t)^{-d / 2}\left(\operatorname{det} A^{0}\right)^{-1 / 2} \exp \left(-x\left(A^{0}\right)^{-1} x / t\right)
$$

- фундаментальное решение усредненного уравнения.

Теорема 1.1 будет получена в качестве следствия теоремы об $L^{2}$-усреднении.

Положим $A^{\varepsilon}(x)=E+B^{\varepsilon}(x), B^{\varepsilon}(x)=B(T(y) \omega), y=\varepsilon^{-1} x$ и рассмотрим семейство задач Коши

$$
\frac{\partial u^{\varepsilon}}{\partial t}=\Delta u^{\varepsilon}+\operatorname{div}\left(B^{\varepsilon} \nabla u^{\varepsilon}\right)=\operatorname{div}\left(A^{\varepsilon} \nabla u^{\varepsilon}\right),\left.\quad u^{\varepsilon}\right|_{t=0}=\varphi \in C_{0}^{\infty}\left(\mathbb{R}^{d}\right) .
$$

ТЕОРема 1.2 (об $L^{2}$-усреднении). Существует постоянная матрица $A^{0}>0$, такая, ито для почти всех $\omega \in \Omega$

$$
\lim _{\varepsilon \rightarrow 0} \int_{\mathbb{R}^{d}}\left|u^{\varepsilon}(x, t, \omega)-u^{0}(x, t)\right|^{2} d x=0 \quad \forall t \geqslant 0,
$$

где $u^{0}(x, t)$ - решение усредненной задачи Коши $\partial u^{0} / \partial t=\operatorname{div}\left(A^{0} \nabla u^{0}\right)$, $\left.u\right|_{t=0}=\varphi$.

Некоторые свойства усреднения были ранее доказаны в [5] при условии, что $\left\langle|b|^{d+\delta}\right\rangle\left\langle\infty,\left\langle|B|^{q}\right\rangle\langle\infty, \delta>0, q=d \quad\right.$ для $d \geqslant 3, \quad q=2+\delta \quad$ для $d=2$.

Указанная формулировка теоремы 1.1 не совсем точна. Дело в том, что при выполнении условий (1.4) имеет место единственность матрицы эффективной 
диффузии (и в этом смысле все точно), но не доказана единственность решения задачи Коши. Последнее само по себе особых проблем не вызывает. Требуется лишь так выбрать решения $\rho(x, t, \omega)$, чтобы обеспечить измеримость по $\omega$. Это получится, если решение $\rho$ определить как подходяший предел решений задач Коши с $B_{N}$ вместо $B$, где $B_{N} \in L^{\infty}(\Omega)$ и $B_{N} \rightarrow B$ в $L^{2}(\Omega)$ при $N \rightarrow \infty$.

В терминах только матрицы $B$ (без привлечения векторного поля $b$ ) удается сделать немного. А именно если $\left\langle|B|^{q}\right\rangle<\infty$ (q определено в (1.9)), то основные теоремы сохраняются, но с одной оговоркой: усредненная матрица, вообще говоря, зависит от указанной выше аппроксимации.

Общий случай $\left\langle|B|^{2}\right\rangle<\infty$ обсуждается в [6]; развитый в этой работе подход очень интересен, хотя не вполне строго обоснован.

3. В дальнейшем придется часто переходить к пределу под знаком интеграла. Напомним соответствуюшие факты. Пусть $Q-$ шар в $\mathbb{R}^{d}$.

ТЕОРема ЛЕБеГА. Если последовательность $w^{\varepsilon} \in L^{1}(Q)$ равностепенно интегрируема и $w^{\varepsilon}(x) \rightarrow w^{0}(x)$ n.в. на $Q$, то $w^{\varepsilon} \rightarrow w^{0}$ в $L^{1}(Q)$.

Известно, что равностепенная интегрируемость эквивалентна слабой компактности в $L^{1}(Q)$.

ЛЕмма 1.3. Пусть $v \in L^{2}(\Omega)$ u $v_{\varepsilon}(x)=v(T(y) \omega), y=\varepsilon^{-1} x$. Если последовательность $u^{\varepsilon}$ ограничена в $L^{2}(Q)$, то произведение $u^{\varepsilon} v_{\varepsilon}$ слабо компактно в $L^{1}(Q)$.

ДокАЗАТЕЛЬство. Последовательность $\left|v_{\varepsilon}\right|^{2}$ равностепенно интегрируема, так как слабо сходится в $L^{1}(Q)$ по эргодической теореме. Отсюда

$$
\int_{K}\left|u^{\varepsilon} v_{\varepsilon}\right| d x \leqslant\left(\int_{K}\left|v_{\varepsilon}\right|^{2} d x\right)^{1 / 2}\left(\int_{Q}\left|u^{\varepsilon}\right|^{2} d x\right)^{1 / 2} \leqslant C\left(\int_{K}\left|v_{\varepsilon}\right|^{2} d x\right)^{1 / 2} \leqslant \delta,
$$

если только мера Лебега $|K|$ достаточно мала.

\section{§2. Вопросы единственности}

1. Ключевая проблема в случае неограниченной матрицы $B-$ это единственность решения. Поясним эту проблему сначала на примере задачи Дирихле в шаре $Q:-\operatorname{div}(\nabla u+B \nabla u)=f \in L^{2}(Q), B \in L^{2}(Q)$. Скажем, что $u \in H_{0}^{1}(Q)$ есть решение этой задачи, если

$$
\int_{Q}(\nabla u \cdot \nabla \varphi+B \nabla u \cdot \nabla \varphi) d x=\int_{Q} f \varphi d x \quad \forall \varphi \in C_{0}^{\infty}(Q) .
$$

Очевидно, что условие $B \in L^{2}(Q)$ есть минимальное требование для того, чтобы это интегральное тождество вообще имело смысл. Существование решения устанавливается без всякого труда. Пусть $B_{N}$ - кососимметричные матрицы, $B_{N} \in L^{\infty}(Q), B_{N} \rightarrow B$ в $L^{2}(Q)$. Тогда решение $u_{N}$ существует и благодаря кососимметричности матрицы $B_{N}$

$$
\int_{Q} \nabla u_{N} \cdot \nabla u_{N} d x=\int_{Q} f u_{N} d x \leqslant\|f\|_{H^{-1}(Q)}\left\|u_{N}\right\|_{H_{0}^{1}(Q)} .
$$


Таким образом, последовательность $u_{N}$ ограничена в $H_{0}^{1}$ и предельным переходом получаем тождество (2.1). Однако единственность ничем не обеспечена. Кроме того, из (2.2) следует только неравенство

$$
\int_{Q}|\nabla u|^{2} d x \leqslant \int_{Q} f u d x
$$

причем равенство («энергетическое равенство») остается под вопросом.

Единственность решения удается доказать только с привлечением векторного поля $b(x)$.

Лемма 2.1. Пусть $b \in L^{2}(Q), \operatorname{div} b=0$. Тогда задача Дирихле $\Delta u+$ $b \cdot \nabla u=f, u \in H_{0}^{1}(Q)$, имеет единственное решение.

ДокаЗАТЕЛЬСТво. Пусть $u$ - решение однородного уравнения. Тогда

$$
\int_{Q} \nabla u \cdot \nabla \varphi d x-\int_{Q} b \cdot \nabla u \varphi d x=0 \quad \forall \varphi \in C_{0}^{\infty}(Q) .
$$

Возьмем срезку

$$
\bar{u}= \begin{cases}u, & \text { если }|u| \leqslant k, \\ \pm k, & \text { если }|u|>k,\end{cases}
$$

и воспользуемся доказанным ниже равенством

$$
\int_{Q} b \cdot \nabla u \bar{u} d x=0 .
$$

Поскольку $b \cdot \nabla u \in L^{1}(Q)$, в тождестве (2.3) можно взять $\varphi=\bar{u}$. В результате $\int_{Q} \nabla u \cdot \nabla \bar{u} d x=0$ и, полагая $k \rightarrow \infty$, получим $\int_{Q}|\nabla u|^{2} d x=0$, т.е. $u=0$ в $H_{0}^{1}$.

Докажем равенство (2.4). Сначала рассмотрим случай $u \geqslant 0$, и пусть $S=$ $\{x \in Q, u>k\}$. Замечаем, что

$$
\begin{aligned}
\int_{Q} b \cdot \nabla \bar{u} \bar{u} d x & =\frac{1}{2} \int_{Q} b \cdot \nabla\left(\bar{u}^{2}\right) d x=0 \quad\left(\text { так как } \bar{u}^{2} \in H_{0}^{1}\right), \\
\int_{Q} b \nabla u \cdot \bar{u} d x & =\int_{Q} b \cdot(\nabla u-\nabla \bar{u}) \bar{u} d x \\
& =k \int_{S} b \cdot(\nabla u-\nabla \bar{u}) d x=k \int_{Q} b \cdot(\nabla u-\nabla \bar{u}) d x=0 .
\end{aligned}
$$

Общий случай легко получается отсюда с помощью разложения $u$ на положительную и отрицательную части.

ПрЕДЛОЖЕНИЕ 2.2. Пусть $b \in L^{2}(Q), \operatorname{div} b=0, b=\operatorname{div} B$, аде $B \in L^{2}(Q)$ - кососимметрическал матрича. Если $u \in H^{1}(Q), \bar{u}-$ срезка, то

$$
\int_{Q} b \cdot \nabla u \bar{u} \varphi d x=-\int_{B} B \nabla u \cdot \nabla \varphi \bar{u} d x \quad \forall \varphi \in C_{0}^{\infty}(Q) .
$$

ДокаЗАтельство. Равенство $b=\operatorname{div} B$ означает по определению, что

$$
\int_{Q} b \cdot \psi d x=-\int_{Q} B \cdot \nabla \psi d x \quad \forall \psi \in\left(C_{0}^{\infty}(Q)\right)^{d} .
$$


Поскольку $b, B \in L^{2}$, это тождество выполнено для $\psi \in\left(H_{0}^{1}\right)^{d}$. Считая функцию $u$ гладкой, возьмем $\psi=\nabla u \bar{u} \varphi$. Тогда $\nabla \psi=\nabla u \times \nabla \bar{u} \varphi+\nabla u \times \nabla \varphi \bar{u}+\bar{u} \varphi \nabla^{2} u$ и $B \nabla u \cdot \nabla \bar{u}=0, B \cdot \nabla^{2} u=0$ п.в. на $Q$ и, значит, равенство (2.5) имеет место. Путем аппроксимации оно распространяется на $u \in H^{1}(Q)$.

2. Перейдем к рассмотрению эллиптических уравнений на вероятностном пространстве $\Omega$. Напомним, что вектор $v=v(\omega)$, заданный на $\Omega, v \in L^{1}(\Omega)$, потенциален (соленоидален), если почти все его реализации $v(x)=v(T(x) \omega)$ потенциальны (соленоидальны) в $\mathbb{R}^{d}$.

Пусть $L_{\mathrm{sol}}^{1}(\Omega)$ - множество всех соленоидальных векторов из $L^{1}(\Omega)$, а $\mathscr{V}_{\text {pot }}^{p}$ - множество всех потенциальных векторов $v$ из $L^{p}(\Omega)(1 \leqslant p \leqslant \infty)$ с нулевым средним, $\langle v\rangle=0$.

Известно, что $\mathscr{V}_{\text {pot }}^{\infty}$ плотно в $\mathscr{V}_{\text {pot }}^{2}$ и для вектора $g \in L^{1}(\Omega)$

$\operatorname{div} g=0 \quad$ (т. е. $\left.g \in L_{\text {sol }}^{1}(\Omega)\right) \Longleftrightarrow \int_{\Omega} g \cdot v d \mu=\langle g \cdot v\rangle=0 \quad \forall v \in \mathscr{V}_{\text {pot }}^{\infty}$.

Пусть $B \in L^{2}(\Omega)$. Существование решения задачи

$$
v \in \mathscr{V}_{\text {pot }}^{2}=V, \quad \operatorname{div}(v+B v)=\operatorname{div} f, \quad f \in L^{2}(\Omega),
$$

устанавливается без труда, например, аппроксимацией.

Скажем, что для нее имеет место свойство единственности, если уравнения $\operatorname{div}(v \pm B v)=0$ не имеют нетривиальных решений. Важно, что при условии единственности для задачи (2.7) найдутся «почти-решения» из $\mathscr{V}_{\text {pot }}^{\infty}$, т. е. для любого $\delta>0$ существуют $v^{\delta} \in \mathscr{V}_{\text {pot }}^{\infty}, f^{\delta} \in L^{2}(\Omega)$, такие, что $\operatorname{div}\left(v^{\delta}+B v^{\delta}\right)=f^{\delta}$, $\lim _{\delta \rightarrow 0}\left\|v-v^{\delta}\right\|_{L^{2}(\Omega)}=0, \lim _{\delta \rightarrow 0}\left\|f-f^{\delta}\right\|_{L^{2}(\Omega)}=0$.

Для доказательства достаточно проверить, что множество $\left\{v+B v, v \in \mathscr{V}_{\text {pot }}^{\infty}\right\}$ плотно в $V^{*}$. Если это не так, то

$$
\exists \varphi \in \mathscr{V}_{\text {pot }}^{2}, \quad 0=\langle\varphi \cdot(v+B v)\rangle=\langle(\varphi-B \varphi) \cdot v\rangle \quad \forall v \in \mathscr{V}_{\text {pot }}^{\infty} .
$$

Из $(2.6)$ следует, что $\operatorname{div}(\varphi-B \varphi)=0$, т. е. $\varphi=0$.

3. Докажем следующее ключевое утверждение.

Лемма 2.3. Условие (1.4) обеспечивает свойство единственности.

ДокаЗАТЕЛЬСТво. Пусть $v \in \mathscr{V}_{\text {pot }}^{2}, \operatorname{div}(v+B v)=0$. Для типичной точки $\omega \in \Omega$ положим $v(x)=v(T(x) \omega), B(x)=B(T(x) \omega)$. Тогда вектор $v(x)$ потенциален, а вектор $v(x)+B(x) v(x)$ соленоидален в $\mathbb{R}^{d}$.

Положим $v_{\varepsilon}(x)=v\left(\varepsilon^{-1} x\right), B^{\varepsilon}(x)=B\left(\varepsilon^{-1} x\right), b^{\varepsilon}(x)=\varepsilon^{-1} b\left(\varepsilon^{-1} x\right)$. Вектор $v_{\varepsilon}$ потенциален в шаре $Q=\{|x|<1\}, v_{\varepsilon} \rightarrow\langle v\rangle=0$ слабо в $L^{2}(Q)$ по эргодической теореме. Поэтому справедливо представление $v_{\varepsilon}=\nabla w_{\varepsilon}$, где $w_{\varepsilon} \rightarrow 0$ слабо в $H^{1}(Q)$. В терминах потенциала $w_{\varepsilon}$ имеем $\Delta w_{\varepsilon}+b^{\varepsilon} \cdot \nabla w_{\varepsilon}=0$ в $Q$. В соответствующем интегральном тождестве можно взять пробную функцию $\varphi \bar{w}_{\varepsilon}$, где $\varphi \in C_{0}^{\infty}(Q)$, а $\bar{w}_{\varepsilon}-$ срезка. Тогда, согласно $(2.5)$,

$$
\int_{Q} \varphi \nabla w_{\varepsilon} \cdot \nabla \bar{w}_{\varepsilon} d x+\int_{Q}\left(\nabla w_{\varepsilon}+B^{\varepsilon} \nabla w_{\varepsilon}\right) \cdot \nabla \varphi \bar{w}_{\varepsilon} d x=0 \quad \forall \varphi \in C_{0}^{\infty}(Q) .
$$

Замечаем, что последовательность $B^{\varepsilon} \nabla w_{\varepsilon}$ слабо компактна в $L^{1}(Q)$ (по лемме 1.3$)$, а $\left|\bar{w}_{\varepsilon}\right| \leqslant k$ по определению срезки. Кроме того, без потери обшности 
считаем, что $\bar{w}_{\varepsilon} \rightarrow 0$ п.в. на $Q$. По теореме Лебега $\left(\nabla w_{\varepsilon}+B_{\varepsilon} \nabla w_{\varepsilon}\right) \cdot \nabla \varphi \bar{w}_{\varepsilon} \rightarrow 0$ в $L^{1}(Q)$ и поэтому

$$
\lim _{\varepsilon \rightarrow 0} \int_{Q} \varphi \nabla w_{\varepsilon} \cdot \nabla \bar{w}_{\varepsilon} d x=0 .
$$

Далее, полагая $L_{\varepsilon}(k)=\left\{x \in Q,\left|w_{\varepsilon}\right|>k\right\}$, имеем

$$
\int_{Q} \varphi \nabla w_{\varepsilon} \cdot \nabla w_{\varepsilon} d x=\int_{Q} \varphi \nabla w_{\varepsilon} \cdot \nabla \bar{w}_{\varepsilon} d x+\int_{L_{\varepsilon}(k)} \varphi \nabla w_{\varepsilon} \cdot \nabla w_{\varepsilon} d x .
$$

Поскольку $w_{\varepsilon}$ ограничена в $L^{1}(Q)$, то $\left|L_{\varepsilon}(k)\right| \leqslant C k^{-1}$. Семейство $\nabla w_{\varepsilon} \cdot \nabla w_{\varepsilon}$ слабо компактно в $L^{1}(Q)$; поэтому второй интеграл справа в $(2.9)$ можно сделать сколь угодно малым за счет выбора $k \geqslant k_{0}$. Отсюда и из $(2.8)$ следует, что

$$
\lim _{\varepsilon \rightarrow 0} \int_{Q} \varphi \nabla w_{\varepsilon} \cdot \nabla w_{\varepsilon} d x=0 \quad \forall \varphi \in C_{0}^{\infty}(Q) .
$$

С другой стороны, по эргодической теореме

$$
\lim _{\varepsilon \rightarrow 0} \int_{Q} \varphi \nabla w_{\varepsilon} \cdot \nabla w_{\varepsilon} d x=\lim _{\varepsilon \rightarrow 0} \int_{Q} \varphi v_{\varepsilon} \cdot v_{\varepsilon} d x=\left\langle v^{2}\right\rangle \int_{Q} \varphi d x>0,
$$

если только $\int_{Q} \varphi d x>0$. Мы получим, что $\left\langle v^{2}\right\rangle=0$. Лемма доказана.

4. Введем теперь собственно задачу на ячейке:

$$
v \in \mathscr{V}_{\text {pot }}^{2}, \quad \operatorname{div}((\xi+v) A)=0, \quad \xi \in \mathbb{R}^{d}, \quad A=E+B .
$$

Если выполняется условие (1.4), то она однозначно разрешима и решение зависит от $\xi$ линейно. Усредненную матрицу определим равенством $\xi A^{0}=\langle(\xi+v) A\rangle$. Для почти-решений имеем

$$
\begin{gathered}
v^{\delta} \in \mathscr{V}_{\text {pot }}^{\infty}, \quad \operatorname{div}\left((\xi+A) v^{\delta}\right)=\operatorname{div} F^{\delta}, \\
\lim _{\delta \rightarrow 0}\left\|v^{\delta}-v\right\|_{L^{2}(\Omega)}=0, \quad \lim _{\delta \rightarrow 0}\left\|F^{\delta}\right\|_{L^{2}(\Omega)}=0 .
\end{gathered}
$$

Положим $q^{\delta}=\left(\xi+v^{\delta}\right) A$. Очевидно, что $\lim _{\delta \rightarrow 0} q^{\delta}=(\xi+v) A$ в $L^{1}(\Omega)$,

$$
\lim _{\delta \rightarrow 0}\left\langle q^{\delta}\right\rangle=\xi A^{0}
$$

Далее, из (2.11) и (2.6) получаем

$$
\begin{aligned}
\left\langle q^{\delta}\right\rangle \cdot \xi & =\left\langle\left(\xi+v^{\delta}\right) A \cdot \xi\right\rangle=\left\langle\left(\xi+v^{\delta}\right) A \cdot\left(\xi+v^{\delta}\right)\right\rangle-\left\langle F^{\delta} \cdot v^{\delta}\right\rangle \\
& =\left\langle\left(\xi+v^{\delta}\right) \cdot\left(\xi+v^{\delta}\right)\right\rangle-\left\langle F^{\delta} \cdot v^{\delta}\right\rangle, \\
\xi A^{0} \xi & =\lim _{\delta \rightarrow 0}\left\langle q^{\delta}\right\rangle \cdot \xi=\langle(\xi+v)(\xi+v)\rangle \geqslant \xi^{2},
\end{aligned}
$$

т. е. $A^{0} \geqslant E$. Матрица $A^{0}$, вообще говоря, несимметрична, но в в усредненном уравнении участвует только симметрическая часть.

5. При выполнении условия (1.4) единственность решения эллиптического уравнения в $\mathbb{R}^{d}$

$$
w \in H^{1}\left(\mathbb{R}^{d}\right), \quad-\operatorname{div}(A \nabla w)+\lambda w=f \in L^{2}\left(\mathbb{R}^{d}\right), \quad \lambda>0,
$$

не доказана. 
Единственность имеет место, если $b \in L^{2}(\Omega), B \in L^{d}(\Omega)$. На доказательстве этого факта останавливаться не будем. Указанное условие обеспечивает и единственность решения задачи Коши $\partial u / \partial t=\operatorname{div}(A(x) \nabla u),\left.u\right|_{t=0}=f \in L^{2}\left(\mathbb{R}^{d}\right)$.

При этом функцию $u(x, t)$ мы называем решением, если

(i) $u(\cdot, t)$ слабо непрерывна как функция от $t \geqslant 0$ со значениями в $L^{2}\left(\mathbb{R}^{d}\right)$;

(ii) $\sup _{t \geqslant 0}\|u(\cdot, t)\|_{L^{2}\left(\mathbb{R}^{d}\right)}<\infty, \nabla u \in L^{2}\left(\mathbb{R}^{d} \times[0, \infty)\right)$;

(iii) преобразование Лапласа

$$
w=w(x, \lambda)=\int_{0}^{\infty} e^{-\lambda t} u(x, t) d t, \quad \lambda>0
$$

(принадлежащее $H^{1}\left(\mathbb{R}^{d}\right)$ в силу (ii)), есть решение уравнения (2.14).

\section{§3. Усреднение}

1. Как и ранее, положим $A^{\varepsilon}(x)=A(T(y) \omega), y=\varepsilon^{-1} x, A=E+B$.

ЛЕмма 3.1. Предположим, что для задачи на ячейке выполнено свойство единственности, например, $b \in L^{2}(\Omega), B \in L^{2}(\Omega)$. Пусть $Q-$ шар $и$

$$
-\operatorname{div}\left(A^{\varepsilon} \nabla u^{\varepsilon}\right)=f^{\varepsilon} \quad \text { в } Q, \quad u^{\varepsilon} \in H^{1}(Q), f^{\varepsilon} \in L^{2}(Q) .
$$

Если $f^{\varepsilon} \rightarrow f, u^{\varepsilon} \rightarrow u^{0}$ слабо в $L^{2}(Q)$ и $H^{1}(Q)$, mо $-\operatorname{div}\left(A^{0} \nabla u^{0}\right)=f$ в $Q$.

Доказательство разобьем на несколько шагов. В общих чертах оно следует классической схеме $[7,1,2]$.

(i) Последовательность векторов $p^{\varepsilon}=A^{\varepsilon} \nabla u^{\varepsilon}$ слабо компактна в $L^{1}(Q)$ (по лемме 1.3), и без потери общности можно считать, что $p^{\varepsilon} \rightarrow p^{0}$ в $L^{1}(Q)$. Переходом к пределу в (3.1) получим тождество

$$
\int_{Q} p^{0} \cdot \nabla h d x=\int_{Q} f h d x, \quad h \in C_{0}^{\infty}(Q),
$$

и требуется доказать равенство $p^{0}=A^{0} \nabla u^{0}$.

(ii) Пусть $v^{\delta}=v^{\delta}(\omega)$ - почти-решения задачи (2.10). Положим $v^{\delta}(x)=$ $v^{\delta}(T(x)), v_{\varepsilon}^{\delta}=v^{\delta}\left(\varepsilon^{-1} x\right)$ и введем аналогичные обозначения для других функций на $\Omega$.

Поскольку $v^{\delta} \in \mathscr{V}_{\text {pot }}^{\infty}$, то при фиксированном $\delta>0$ вектор $v_{\varepsilon}^{\delta}$ потенциален в $Q,\left|v_{\varepsilon}^{\delta}\right| \leqslant l(\delta), v_{\varepsilon}^{\delta} \rightarrow 0$ слабо в $L^{2}(Q)$. Отсюда следует представление

$$
v_{\varepsilon}^{\delta}=\nabla w^{\varepsilon}, \quad w^{\varepsilon} \in W^{1, \infty}(Q), \quad \lim _{\varepsilon \rightarrow 0}\left\|w^{\varepsilon}\right\|_{L^{\infty}(Q)}=0,
$$

и при этом $\operatorname{div}\left[\left(\xi+\nabla w^{\varepsilon}\right) A^{\varepsilon}\right]=\operatorname{div} F_{\varepsilon}^{\delta}$ в $Q,\left(\xi+\nabla w^{\varepsilon}\right) A^{\varepsilon}=q_{\varepsilon}^{\delta}$, где $q^{\delta}=\left(\xi+v^{\delta}\right) A$.

(iii) Итак, имеем два интегральных тождества

$$
\begin{aligned}
\int_{Q} A^{\varepsilon} \nabla u^{\varepsilon} \cdot \nabla h & =\int_{Q} f^{\varepsilon} h d x \\
\int_{Q}\left(\xi+\nabla w^{\varepsilon}\right) A^{\varepsilon} \cdot \nabla h d x & =\int_{Q} F_{\varepsilon}^{\delta} \cdot \nabla h d x, \quad h \in C_{0}^{\infty}(Q) .
\end{aligned}
$$


Возьмем в первом из них $h=\varphi\left(w^{\varepsilon}+\xi \cdot x\right)$, а во втором $h=\varphi u^{\varepsilon}, \varphi \in C_{0}^{\infty}(Q)$. Это можно сделать в силу того, что $A^{\varepsilon} \in L^{2}(Q), w^{\varepsilon} \in L^{\infty}(Q)$. В результате получаем

$$
\begin{aligned}
\int_{Q} f^{\varepsilon}\left(\xi \cdot x+w^{\varepsilon}\right) \varphi d x- & \int_{Q}\left(\xi \cdot x+w^{\varepsilon}\right) p^{\varepsilon} \cdot \nabla \varphi d x \\
& =\int_{Q} F_{\varepsilon}^{\delta} \cdot \nabla\left(\varphi u^{\varepsilon}\right) d x-\int_{Q} u^{\varepsilon} q_{\varepsilon}^{\delta} \cdot \nabla \varphi d x=J_{\varepsilon},
\end{aligned}
$$

и требуется перейти в этом равенстве к пределу сначала при $\varepsilon \rightarrow 0$, а затем при $\delta \rightarrow 0$. Рассмотрим левую часть равенства (3.4). В силу (3.3) к последовательности $\left(\xi \cdot x+w^{\varepsilon}\right) p^{\varepsilon} \cdot \nabla \varphi$ применима теорема Лебега. Поэтому предел левой части равен

$$
J=\int_{Q} f \xi \cdot x \varphi d x-\int_{Q} \xi \cdot x p^{0} \cdot \nabla \varphi d x .
$$

Кроме того, взяв $h=\varphi \xi \cdot x$ в тождестве $(3.2)$, получим соотношение

$$
\int_{Q} \xi \cdot x p^{0} \cdot \nabla \varphi d x+\int_{Q} p^{0} \cdot \xi \varphi d x=\int_{Q} f \xi \cdot x \varphi d x
$$

из которого ясно, что

$$
J=\int_{Q} p^{0} \cdot \xi \varphi d x
$$

Изучим теперь предел правой части равенства (3.4). Так как $q^{\delta} \in L^{2}(\Omega)$, то $q_{\varepsilon}^{\delta} \rightarrow\left\langle q^{\delta}\right\rangle$ слабо в $L^{2}(Q)$. Поскольку $u^{\varepsilon} \rightarrow u^{0}$ сильно в $L^{2}(Q)$,

$$
\lim _{\varepsilon \rightarrow 0} \int_{\mathbb{R}^{d}} u^{\varepsilon} q_{\varepsilon}^{\delta} \cdot \nabla \varphi d x=\int_{\mathbb{R}^{d}} u^{0}\left\langle q^{\delta}\right\rangle \cdot \nabla \varphi d x .
$$

Последовательность $\nabla\left(\varphi u^{\varepsilon}\right)$ ограничена в $L^{2}(Q)$, и по эргодической теореме

$$
\lim _{\varepsilon \rightarrow 0}\left|\int_{Q} F_{\varepsilon}^{\delta} \cdot \nabla\left(\varphi u^{\varepsilon}\right) d x\right| \leqslant C \lim _{\varepsilon \rightarrow 0} \int_{Q}\left|F_{\varepsilon}^{\delta}\right|^{2} d x=C|Q|\left\langle\left|F^{\delta}\right|^{2}\right\rangle .
$$

Учитывая (3.6), (2.12), (2.13), заключаем, что

$$
\lim _{\delta \rightarrow 0} \lim _{\varepsilon \rightarrow 0} J_{\varepsilon}=-\int_{\mathbb{R}^{d}} u^{0}\langle q\rangle \cdot \nabla \varphi=-\int_{Q} \xi A^{0} \cdot \varphi u^{0}=\int_{Q} A^{0} \nabla u^{0} \cdot \xi \varphi d x .
$$

Сравнивая этот результат с (3.5), получим искомое соотношение $p^{0}=A^{0} \nabla u^{0}$. Лемма доказана.

2. Перейдем к усреднению задачи Коши

$$
\frac{\partial u^{\varepsilon}}{\partial t}=\operatorname{div}\left(A^{\varepsilon} \nabla u^{\varepsilon}\right),\left.\quad u^{\varepsilon}\right|_{t=0}=f^{\varepsilon}, \quad f^{\varepsilon} \rightarrow f \text { слабо в } L^{2}\left(\mathbb{R}^{d}\right) .
$$

Ввиду возможной неединственности требуется сначала уточнить, о каких решениях идет речь. При фиксированном $\varepsilon>0$ мы определим решение с помошью аппроксимаций. Пусть $B_{N} \in L^{\infty}(\Omega), B_{N}$ кососимметричны и $B_{N} \rightarrow B$ в $L^{2}(\Omega)$ 
при $N \rightarrow \infty$. Тогда $B_{N}(x) \rightarrow B(x)$ в $L_{\text {loc }}^{2}\left(\mathbb{R}^{d}\right)$ (для п.в. $\omega$ ), и возникает аппроксимация задачи (3.7) в виде $\partial u_{N}^{\varepsilon} / \partial t=\operatorname{div}\left(A_{N}^{\varepsilon} \nabla u_{N}^{\varepsilon}\right),\left.u_{N}^{\varepsilon}\right|_{t=0}=f^{\varepsilon}$.

Рассмотрим $u_{N}^{\varepsilon}(x, t)$ как функции аргумента $t \geqslant 0$ со значениями в $L^{2}\left(\mathbb{R}^{d}\right)$ и докажем, что это семейство слабо равностепенно непрерывно на $[0, \infty)$.

Действительно, в силу энергетической оценки

$$
\int_{\mathbb{R}^{d}}\left|u_{N}^{\varepsilon}(x, t)\right|^{2} d t+\int_{0}^{t} \int_{\mathbb{R}^{d}}\left|\nabla u_{N}^{\varepsilon}\right|^{2} d x d t=\int_{\mathbb{R}^{d}}\left|f^{\varepsilon}\right|^{2} d x \leqslant l^{2}<\infty
$$

достаточно проверить, что для любой функции $\varphi \in C_{0}^{\infty}\left(\mathbb{R}^{d}\right)$ семейство функций $\alpha_{N}^{\varepsilon}=\int_{\mathbb{R}^{d}} u_{N}^{\varepsilon} \varphi d x$ равностепенно непрерывно на $[0, \infty)$. Пусть $\operatorname{supp} \varphi \subset Q$, где $Q$ - шар. Тогда

$$
\begin{aligned}
& \left|\alpha_{N}^{\varepsilon}\left(t_{2}\right)-\alpha_{N}^{\varepsilon}\left(t_{1}\right)\right|=\left|\int_{t_{1}}^{t_{2}} \int_{\mathbb{R}^{d}} A_{N}^{\varepsilon} \nabla u_{N}^{\varepsilon} \cdot \nabla \varphi d x d t\right| \\
& \quad \leqslant\left|t_{2}-t_{1}\right|^{1 / 2} \sup |\nabla \varphi|\left(\int_{Q}\left|A_{N}^{\varepsilon}\right|^{2} d x\right)^{1 / 2}\left(\int_{t_{1}}^{t_{2}} \int_{\mathbb{R}^{d}}\left|\nabla u_{N}^{\varepsilon}\right|^{2} d x d t\right)^{1 / 2} .
\end{aligned}
$$

Так как $\int_{Q}\left|A_{N}^{\varepsilon}\right|^{2} d x \leqslant C_{0}(Q)$, где $C_{0}$ не зависит от $\varepsilon, N$, то

$$
\left|\alpha_{N}^{\varepsilon}\left(t_{2}\right)-\alpha_{N}^{\varepsilon}\left(t_{1}\right)\right| \leqslant l C_{1}(\varphi, Q)\left|t_{2}-t_{1}\right|^{1 / 2},
$$

и утверждение доказано.

Теперь можно определить решение $u^{\varepsilon}$ уравнения (3.7) (при фиксированном $\varepsilon$ ) как предел подпоследовательности $u_{N^{\prime}}^{\varepsilon}$ при $N^{\prime} \rightarrow \infty$. Очевидно, что решения $u^{\varepsilon}$ останутся слабо равностепенно непрерывными на $[0, \infty)$. Займемся предельным переходом при $\varepsilon \rightarrow 0$. Без потери общности полагаем, что при $\varepsilon \rightarrow 0$

$$
u^{\varepsilon}(\cdot, t) \rightarrow u^{0}(\cdot, t) \text { слабо в } L^{2}\left(\mathbb{R}^{d}\right) \quad \forall t \geqslant 0 .
$$

Для преобразований Лапласа $w^{\varepsilon}$ имеем $-\operatorname{div}\left(A^{\varepsilon} \nabla w^{\varepsilon}\right)+\lambda w^{\varepsilon}=f^{\varepsilon}$, а из (3.9) и получающегося из (3.8) энергетического неравенства следует, что

$$
w^{\varepsilon} \rightarrow w^{0}=\int_{0}^{\infty} e^{-\lambda t} u^{0}(x, t) d t \quad \text { слабо в } H^{1}\left(\mathbb{R}^{d}\right) .
$$

Тогда, согласно лемме $3.1,-\operatorname{div}\left(A^{0} \nabla w^{0}\right)+\lambda w^{0}=f$. Отсюда легко заключаем, что в ситуачии задачи (3.7) решения слабо сходятся (см. (3.9)) к решению усредненной задачи с предельным начальным значением.

Задача Коши (1.7) получается из (3.7), когда $f^{\varepsilon}=\varphi$ не зависит от $\varepsilon$. Покажем, что в этом случае имеет место $L^{2}$-сходимость (1.8). Для этого наряду с (1.7) рассмотрим сопряженную задачу $\partial v^{\varepsilon} / \partial t=\operatorname{div}\left(\nabla v^{\varepsilon} A^{\varepsilon}\right),\left.v^{\varepsilon}\right|_{t=0}=g^{\varepsilon}$. Решения этих задач будут связаны формулой Грина

$$
\int_{\mathbb{R}^{d}} u^{\varepsilon}\left(x, t_{0}\right) g^{\varepsilon}(x) d x=\int_{\mathbb{R}^{d}} v^{\varepsilon}\left(x, t_{0}\right) \varphi(x) d x,
$$

если их определить путем одновременной аппроксимации. Положим $g^{\varepsilon}=u^{\varepsilon}\left(x, t_{0}\right)$ $-u^{0}\left(x, t_{0}\right)$. Согласно предыдущему, $v^{\varepsilon}\left(x, t_{0}\right) \rightarrow 0$ слабо в $L^{2}\left(\mathbb{R}^{d}\right)$ и из $(3.10)$ получаем соотношение

$$
\lim _{\varepsilon \rightarrow 0} \int_{\mathbb{R}^{d}} u^{\varepsilon}\left(x, t_{0}\right)\left[u^{\varepsilon}\left(x, t_{0}\right)-u^{0}\left(x, t_{0}\right)\right] d x=0,
$$


из которого следует сильная сходимость (1.8).

Итак, для почти каждого $\omega \in \Omega$ мы построили решение $u^{\varepsilon}(x, t, \omega)$ задачи Коши (1.7) и доказали теорему об $L^{2}$-усреднении. В случае единственности решения к этому добавить нечего - функция $u^{\varepsilon}$ будет измерима на $\mathbb{R}^{d} \times \Omega \times[0, \infty)$.

В случае неединственности измеримость по $\omega \in \Omega$ ниоткуда не следует. В связи с этим нужно слегка изменить процесс построения решения.

Снова берем решения $u_{N}^{\varepsilon}(x, t, \omega)$ ( $\varepsilon>0$ пока фиксировано), но рассматриваем их как функции аргумента $t \geqslant 0$ со значениями в $L^{2}\left(\mathbb{R}^{d} \times \Omega\right)$. Это семейство слабо равностепенно непрерывно, и мы определяем $u^{\varepsilon}$ как слабый предел подпоследовательности $u_{N^{\prime}}^{\varepsilon}$ при $N^{\prime} \rightarrow \infty$ для любого $t \geqslant 0$. Легко понять, что для почти всех $\omega \in \Omega$ семейство $u^{\varepsilon}(x, t, \omega)$ слабо равностепенно непрерывно в $L^{2}\left(\mathbb{R}^{d}\right)$.

Остается проверить, что $u^{\varepsilon}(x, t, \omega)$ есть решение предельного уравнения. Для этого берем преобразование Лапласа $w_{N}^{\varepsilon}(x, \lambda, \omega)$ и интегральное тождество

$$
\begin{gathered}
\int_{\Omega} \int_{\mathbb{R}^{d}} A_{N}^{\varepsilon} \nabla w_{N}^{\varepsilon} \cdot \nabla \varphi h d x d \mu+\lambda \int_{\Omega} \int_{\mathbb{R}^{d}} w_{N}^{\varepsilon} \varphi h d x d \mu=\int_{\Omega} \int_{\mathbb{R}^{d}} f^{\varepsilon} \varphi h d x d \mu \\
\left(\varphi \in C_{0}^{\infty}\left(\mathbb{R}^{d}\right), h \in L^{\infty}(\Omega)\right),
\end{gathered}
$$

в котором нетрудно перейти к пределу при $N^{\prime} \rightarrow \infty$, используя энергетическую оценку (3.8). В свою очередь, из предельного тождества следует, что

$$
\int_{\mathbb{R}^{d}} A^{\varepsilon} \nabla w^{\varepsilon} \cdot \nabla \varphi d x+\lambda \int_{\mathbb{R}^{d}} w^{\varepsilon} \varphi d x=\int_{\mathbb{R}^{d}} f \varphi d x,
$$

т. е. $u^{\varepsilon}$ есть решение уравнения диффузии.

\section{§4. Связь между усреднением и диффузионным поведением}

В некоторых случаях указанная в заголовке связь устанавливается чрезвычайно просто. Все зависит от того, в какой форме доказаны свойства усреднения. Пусть $u^{\varepsilon}(x, t, \omega)$ - решение задачи Коши (1.7) и для п.в. $\omega \in \Omega$ имеет место поточечная сходимость:

$$
\lim _{\varepsilon \rightarrow 0} \sup _{|x| \leqslant R}\left|u^{\varepsilon}(x, t, \omega)-u^{0}(x, t)\right|=0 \quad(\forall t>0, R>0) .
$$

Плотность $\rho_{\varepsilon}(x, t, \omega)=\varepsilon^{-d} \rho\left(\varepsilon^{-1} x, \varepsilon^{-2} t, \omega\right)$ есть решение задачи Коши

$$
\frac{\partial \rho_{\varepsilon}}{\partial t}=\operatorname{div}\left(\nabla \rho_{\varepsilon} A_{\varepsilon}\right),\left.\quad \rho_{\varepsilon}\right|_{t=0}=\varepsilon^{-d} \chi\left(\varepsilon^{-1} x\right)=\chi_{\varepsilon}(x) .
$$

По формуле Грина

$$
\begin{array}{r}
\int_{\mathbb{R}^{d}}\left[\rho_{\varepsilon}(x, t, \omega)-K_{0}(x, t)\right] \varphi(x) d x=\int_{\mathbb{R}^{d}} u^{\varepsilon}(x, t, \omega) \chi_{\varepsilon}(x) d x-u^{0}(0, t) \\
=\int_{\mathbb{R}^{d}}\left(u^{\varepsilon}(x, t, \omega)-u^{0}(x, t)\right) \chi_{\varepsilon}(x) d x+o(1) \quad \text { при } \varepsilon \rightarrow 0,
\end{array}
$$


поскольку $\chi_{\varepsilon}$ является дельтаобразной последовательностью. Из (4.1) и оценки $\left|u^{\varepsilon}(x, t, \omega)\right| \leqslant \sup |\varphi|$ ясно, что первое слагаемое в (4.2) также есть $o(1)$. Получаем, что для почти всех $\omega$

$$
\lim _{\varepsilon \rightarrow 0} \int_{\mathbb{R}^{d}}\left[\varepsilon^{-d} \rho\left(\varepsilon^{-1} x, \varepsilon^{-2} t, \omega\right)-K_{0}(x, t)\right] \varphi(x) d x=0 \quad \forall \varphi \in C_{0}^{\infty}\left(\mathbb{R}^{d}\right), t \geqslant 0,
$$

т. е. устанавливаем индивидуальную и.n.m. Очевидно, это более сильное утверждение, чем теорема 1.1. Часто $L^{2}$-сходимость $(1.8)$ влечет за собой поточечную сходимость (4.1). Например, это так, если $B \in L^{\infty}(\Omega)$, поскольку в этом случае решения $u^{\varepsilon}(x, t, \omega)(t>0)$ гёльдеровы по $x$ равномерно по $\varepsilon$.

В наших условиях нельзя гарантировать даже непрерывности функции $u^{\varepsilon}(x, t, \omega)$ по $x$ при фиксированном $\varepsilon>0$, и индивидуальная ц.п.т. под вопросом.

Покажем, что теорема об $L^{2}$-усреднении влечет за собой ц.п.т. в среднем. Положим

$$
\Phi_{\varepsilon}(x, t)=\left(\int_{\Omega}\left|u^{\varepsilon}(x, t, \omega)-u^{0}(x, t)\right|^{2} d \mu\right)^{1 / 2},
$$

где $u^{\varepsilon}(x, t, \omega)$ - решение задачи Коши (1.7).

Лемма 4.1. Семейство $\Phi_{\varepsilon}$ равномерно липшичево на $\mathbb{R}^{d}$, а именно

$$
\left|\Phi_{\varepsilon}\left(x_{1}, t\right)-\Phi_{\varepsilon}\left(x_{2}, t\right)\right| \leqslant l\left|x_{1}-x_{2}\right|, \quad l=2 \sup |\nabla \varphi| .
$$

ДокАЗАтЕльство. Сначала предположим, что решения $u^{\varepsilon}(x, t, \omega)$ непрерывны по $x \in \mathbb{R}^{d}$ и для них справедлив обычный принцип максимума. Введем обозначение $u^{\varepsilon}(x, t, \omega)=u^{\varepsilon}(x, t, \omega, \varphi)$ и более широкое семейство решений

$$
\bar{u}^{\varepsilon}(x, y, t, \omega)=u^{\varepsilon}\left(x, t, \omega, \varphi_{y}\right), \quad \varphi_{y}(\cdot)=\varphi(\cdot+y),
$$

так что $y \in \mathbb{R}^{d}$ играет роль параметра. Легко проверяется, что

$$
u^{\varepsilon}(x, t, \omega)=\bar{u}^{\varepsilon}(x, 0, t, \omega)=\bar{u}^{\varepsilon}(0, x, t, T(-x) \omega) .
$$

Мера $\mu$ инвариантна относительно преобразования $T(x)$ и поэтому

$$
\begin{aligned}
\Phi_{\varepsilon}^{2}(x, t) & =\int_{\Omega}\left|\bar{u}^{\varepsilon}(0, x, t, T(-x) \omega)-u^{0}(x, t)\right|^{2} d \mu \\
& =\int_{\Omega}\left|\bar{u}^{\varepsilon}(0, x, t, \omega)-u^{0}(x, t)\right|^{2} d \mu .
\end{aligned}
$$

Но $\bar{u}^{\varepsilon}\left(0, x_{1}, t, \omega\right)$ и $\bar{u}^{\varepsilon}\left(0, x_{2}, t, \omega\right)$ - это значения в нуле решений одного и того же уравнения с различными начальными значениями. По принципу максимума $\left|\bar{u}^{\varepsilon}\left(0, x_{1}, t, \omega\right)-\bar{u}^{\varepsilon}\left(0, x_{2}, t, \omega\right)\right| \leqslant \sup \left|\varphi\left(z+x_{1}\right)-\varphi\left(z+x_{2}\right)\right| \leqslant\left|x_{1}-x_{2}\right| \sup |\nabla \varphi|$.

Поскольку аналогично оценивается $\left|u^{0}\left(x_{1}, t\right)-u^{0}\left(x_{2}, t\right)\right|$, то неравенство (4.3) доказано. Остается избавиться от предположения, что решение задачи (1.7) непрерывно по $x$. Рассмотрим аппроксимирующие решения $u_{N}^{\varepsilon}$, для которых указанные свойства непрерывности и принцип максимума, очевидно, выполняются. Для них из (4.3) имеем

$$
\left\|u_{N}^{\varepsilon}\left(x_{1}, t, \cdot\right)-u^{0}\left(x_{1}, t\right)\right\|_{L^{2}(\Omega)}-\left\|u_{N}^{\varepsilon}\left(x_{2}, t, \cdot\right)-u^{0}\left(x_{2}, t\right)\right\|_{L^{2}(\Omega)} \leqslant l\left|x_{1}-x_{2}\right| .
$$


Обозначим через $v_{N}^{\varepsilon}$ последовательность выпуклых комбинаций функций $u_{N}^{\varepsilon}$, таких, что

$$
v_{N}^{\varepsilon}(x, t, \omega) \rightarrow u^{\varepsilon}(x, t, \omega) \text { в } L^{2}(\Omega) \text { при } N \rightarrow \infty
$$

для почти всех $x \in \mathbb{R}^{d}$ и фиксированных $\varepsilon>0, t>0$. Важно заметить, что неравенство (4.4) сохраняется, если заменить $u_{N}^{\varepsilon}$ на $v_{N}^{\varepsilon}$. Поэтому и для решения $u^{\varepsilon}(x, t, \omega)$ оно выполнено (для почти всех $x_{1}, x_{2} \in \mathbb{R}^{d}$ ). Лемма доказана.

Теперь из $L^{2}$-сходимости (1.8) по теореме Лебега следует, что

$$
\lim _{\varepsilon \rightarrow 0} \int_{\Omega} \int_{\mathbb{R}^{d}}\left|u^{\varepsilon}(x, t, \omega)-u^{0}(x, t)\right|^{2} d x d \mu=\lim _{\varepsilon \rightarrow 0} \int_{\mathbb{R}^{d}} \Phi_{\varepsilon}^{2}(x, t) d x=0, \quad t \geqslant 0,
$$

и это вместе с оценкой (4.3) означает, что

$$
\lim _{\varepsilon \rightarrow 0} \Phi_{\varepsilon}(x, t)=0 \text { равномерно на компактах из } \mathbb{R}^{d} .
$$

Из (4.2) (с точностью до о(1)) имеем

$$
\begin{aligned}
& \int_{\Omega}\left|\int_{\mathbb{R}^{d}}\left[\rho_{\varepsilon}(x, t, \omega)-K_{0}(x, t)\right] \varphi(x) d x\right| d \mu \\
& \quad \leqslant \int_{\Omega} \int_{\mathbb{R}^{d}}\left|u^{\varepsilon}(x, t, \omega)-u^{0}(x, t)\right| \chi_{\varepsilon}(x) d x d \mu \\
& \quad \leqslant \int_{\Omega}\left(\int_{\mathbb{R}^{d}}\left|u^{\varepsilon}(x, t, \omega)-u^{0}(x, t)\right|^{2} \chi_{\varepsilon}(x) d x\right)^{1 / 2} d \mu \\
& \quad \leqslant\left(\int_{\mathbb{R}^{d}} \Phi_{\varepsilon}^{2}(x, t) \chi_{\varepsilon}(x) d x\right)^{1 / 2} \rightarrow 0
\end{aligned}
$$

в силу (4.5) и оценки $0 \leqslant \Phi_{\varepsilon} \leqslant 2 \sup |\varphi|$. Теорема 1.1 доказана.

\section{ЛитеРАтУРА}

1. Жиков В. В., Козлов С. М., Олейник О. А. Усреднение дифференциальных операторов. Наука, М., 1993

2. Jikov V., Koslov S., Oleinik O. Homogenization of differential operators and integral functionals. Springer-Verlag, Berlin, 1994.

3. Matheron G., de Marsity G. Is transport in porous media always diffusive? A counterexample. Water Resource Res., 16, 901-917 (1980).

4. Жиков В. В. Замечания к проблеме остаточной диффузии. УМН, $\mathbf{4 4}$, №6, $155-156$ (1989).

5. Avelaneda M., Majda A. J. An integral representation and bounds on the effective diffusivity in passive advection by laminar and turbulent flows. Commun. Math. Phys., 138, 339-391 (1991).

6. Fannjiang A., Papanicolaou G. C. Diffusion in turbulence, Probability Theory and Related Fields, No. 105, 279-334 (1996).

7. Tartar L. Homogénéisation. Cours Peccot au Collège de France, Paris, 1977. 